\title{
Primary CNS Melanoma: A Case Report with Review of the Literature
} Rana Shaker AL-Zaidi ${ }^{*}$

Department of pathology and laboratory medicine, King Faisal Hospital, Prince Majed Ibn Abdulaziz Road, AL Maabda, Makkah, Kingdom of Saudi Arabia

DOI: $10.36348 /$ sjpm.2020.v05i10.001 | Received: 19.09.2020 | Accepted: 27.09.2020 | Published: 03.10 .2020

*Corresponding author: Rana Shaker AL-Zaidi

\section{Abstract}

Primary central nervous system (CNS) melanomas are rare tumors arise from precursor leptomeningeal melanocytes that are scattered throughout the arachnoid membranes. The nodular form of primary CNS melanoma manifests as a solitary, discrete neoplasm with a predilection for the spinal cord and posterior fossa. Around 100 cases of primary CNS melanoma reported in the literature. Here, we report the first case in Saudi Arabia of a 55-year-old man who presented with nausea, vomiting, and sudden loss of consciousness. CT brain with contrast showed a left temporal lesion, which was resected and histologically proved to be primary CNS melanoma.

Keywords: Primary central nervous system, melanoma, meningeal.

Copyright @ 2020: This is an open-access article distributed under the terms of the Creative Commons Attribution license which permits unrestricted use, distribution, and reproduction in any medium for non-commercial use (NonCommercial, or CC-BY-NC) provided the original author and sources are credited.

\section{INTRODUCTION}

Primary central nervous system (CNS) melanomas are rare tumors that represent $1 \%$ of all cases of melanoma and $0.07 \%$ of all brain tumors $[1,2]$. Around 100 cases of primary CNS melanoma reported in the literature. They arise from leptomeningeal melanocytes that are scattered throughout the arachnoid membranes. The first case of primary CNS melanoma was described by Virchow, in 1859 [3]. The chief diagnostic dilemma is to differentiate between primary CNS melanoma \& metastatic CNS melanoma. Primary tumors tend to solitary with involvement of the leptomeninges. Metastatic CNS melanoma, in the other hand, are often multiple and involve the CNS parenchyma. The diagnosis of primary CNS melanoma should be made in the proper clinical context and after the exclusion of any possible primary extracranial sites of origin such as skin, scalp, mucosal surfaces, orbit, and adrenal glands. Pappenheim et al reviewed 45 cases before 196 [4]. Since this year and after a thorough search through the literature, we find out thirty-four accessible reported cases of primary intracranial melanoma. Primary spinal melanoma cases are not included in this review.

\section{CASE PRESENTATION}

A 55 years old male, right-handed patient presented to the emergency department complaining of nausea and vomiting for two days. He gave a history of headache and dysarthria for more than 6 months duration. He developed sudden loss of consciousness and fits, and he was comatose upon arrival to the hospital. He had dilated pupil in the left eye with mild reaction to light and normal right pupil in size and reaction to light. His seizure was controlled with antiepileptic medications, under urgent condition. He underwent CT brain with IV contrast for urgent diagnosis and early intervention. It showed an evidence of a solitary left temporal parenchymal hyperdense lesion about $(4 \times 3 \mathrm{~cm})$. It showed strong enhancement post IV contrast with hyper vascularity. It is surrounded by another mild hyperdense area; possibly hemorrhage with marked white matter edema causing right deviation of midline structures and uncal herniation (Fig. 1, 2).

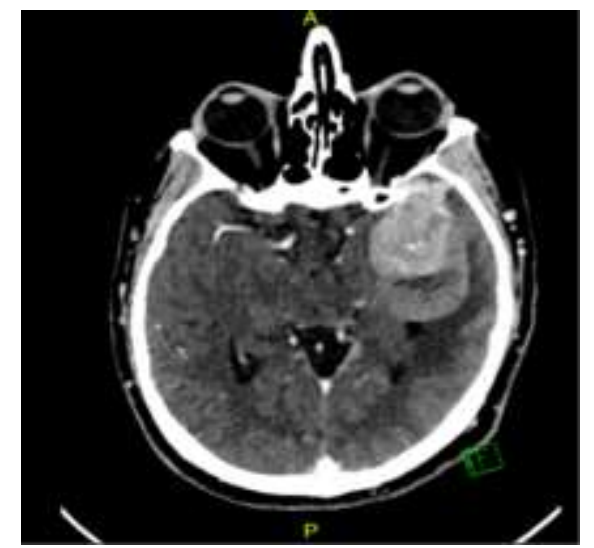

Fig-1: CT brain with IV contrast, axial view. Left temporal lesion $(4 X 5 \mathrm{~cm})$ showing dural attachment $\&$ two different densities. Uncal herniation is noted (Original) 


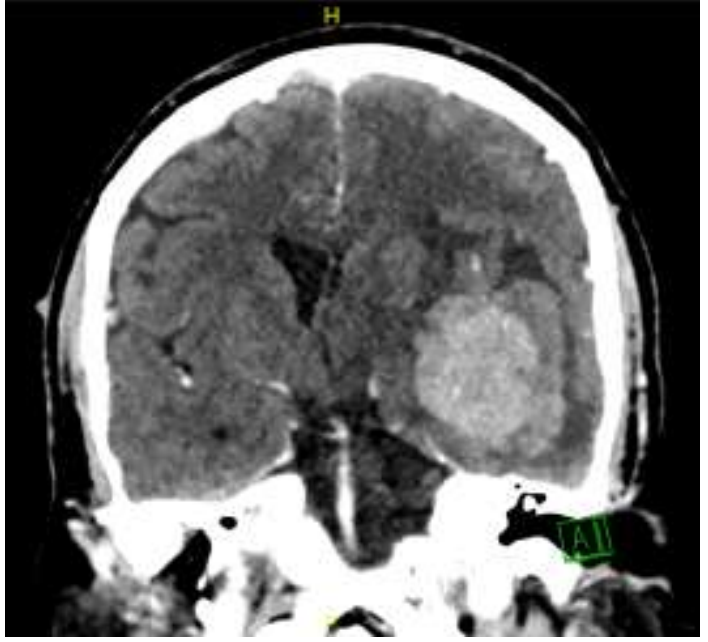

Fig-2: Coronal CT brain with IV contract. Left temporal lesion with significant midline shift and compression of the lateral ventricle (Original)

\section{Pathological findings}

Grossly, the excised tumor consisted of multiple irregular fragments of dark red to brown tissue, measuring $6 \times 3 \times 1 \mathrm{~cm}$, in aggregate. Serial sectioning revealed a heterogeneous tan to dark brown soft surface. Histological sections showed a cellular neoplastic growth comprised of solid sheets and loose nests of atypical cells with focal perivascular growth pattern (papillae/pseudorosette formation)

(Fig. 3,4,5). Individual tumor cells show variable morphology with epithelioid and spindled cells. The cytoplasm is scant to ample, pale amphophilic to dense eosinophilic. The nuclei are moderately pleomorphic, large, round to oval, with vesicular to coarse chromatin pattern and thick nuclear membrane. Large prominent eosinophilic nucleoli and intranuclear pseudoinclusions are present (Fig.6,7). Biand multinucleated tumor cells are occasionally seen. Coarse dark brown melanin pigment is seen in the cytoplasm of many tumor cells as well as in melanophages. Mitotic figures were infrequent (12/HPF). Zones of coagulative-type tumor necrosis and areas of hemorrhage are noted. The tumor is invading the adjacent cortical brain tissue.

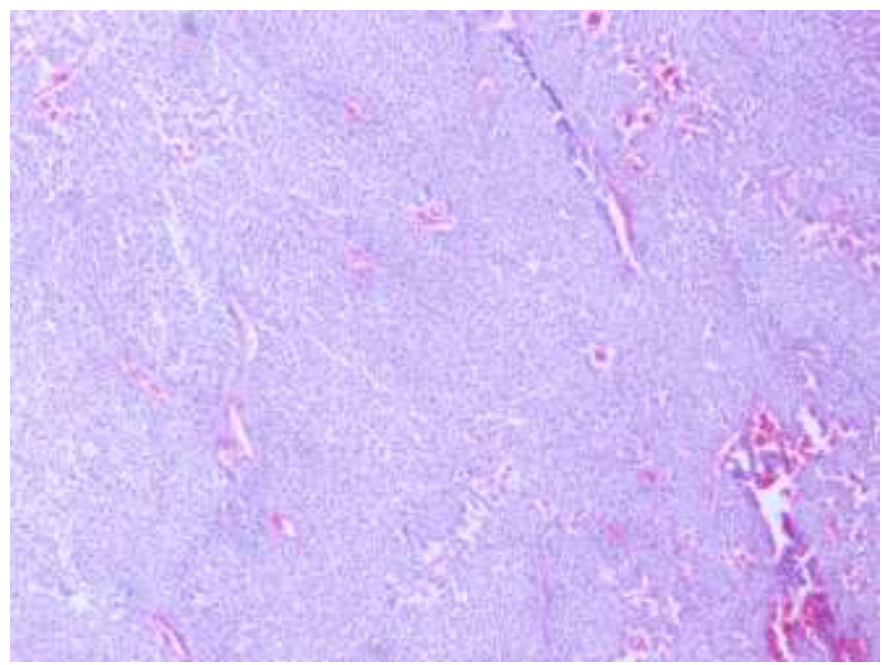

Fig-3: Primary central nervous system melanoma showing solid sheets of spindled to epithelioid cells (hematoxylin-eosin, original magnification X40)

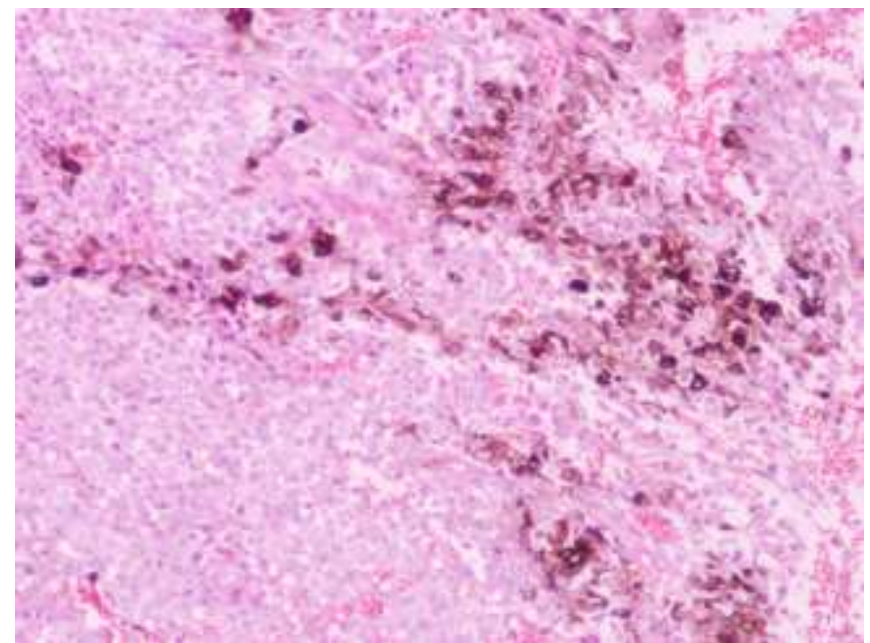

Fig-4: Tumor comprised of loosely cohesive sheets of epithelioid cells with obvious coarse granular melanin pigment (hematoxylin-eosin, original magnification X100). 


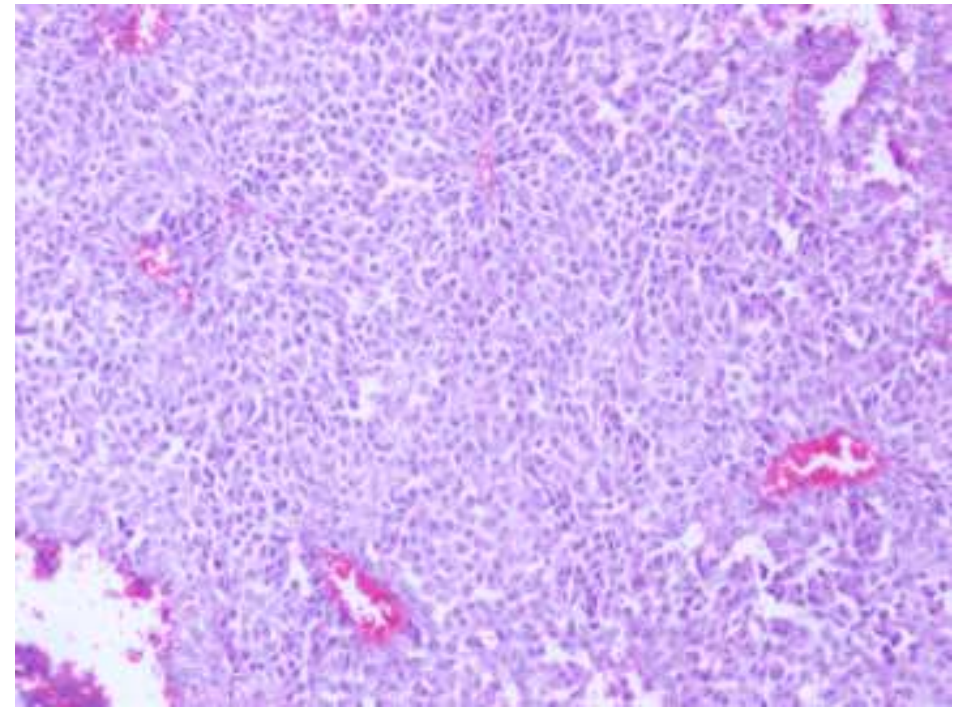

Fig-5: Sheets of epithelioid tumor cells with perivascular condensation (hematoxylin-eosin, original magnification X100)

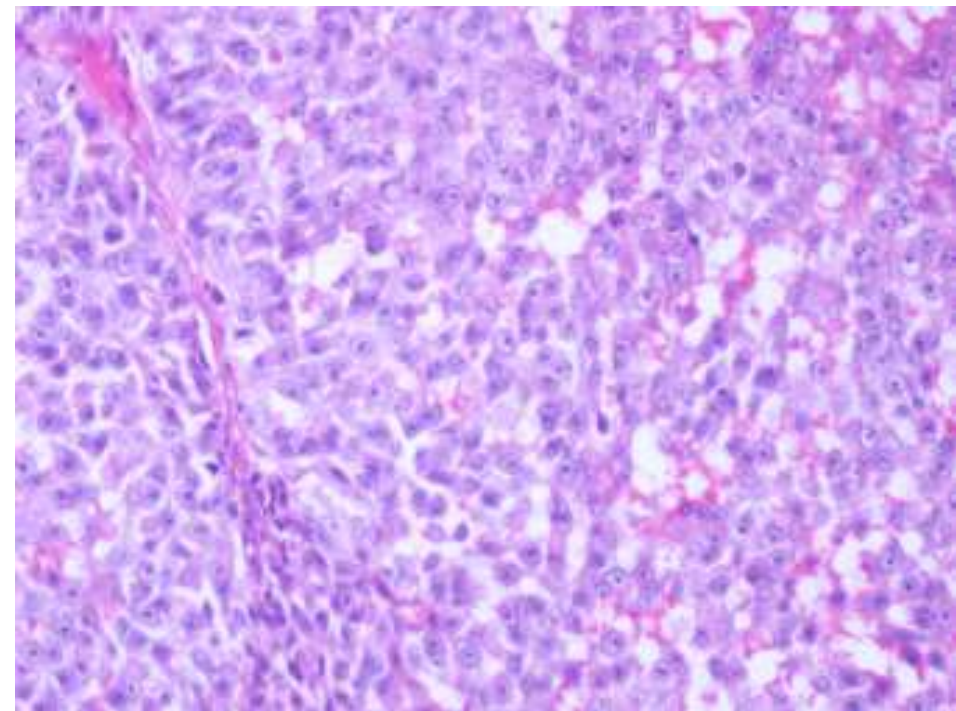

Fig-6: Primary central nervous system melanoma showing loosely cohesive sheets of epithelioid cells with prominent nucleoli and occasional intranuclear pseudoinclusion (hematoxylin-eosin, original magnification X200)

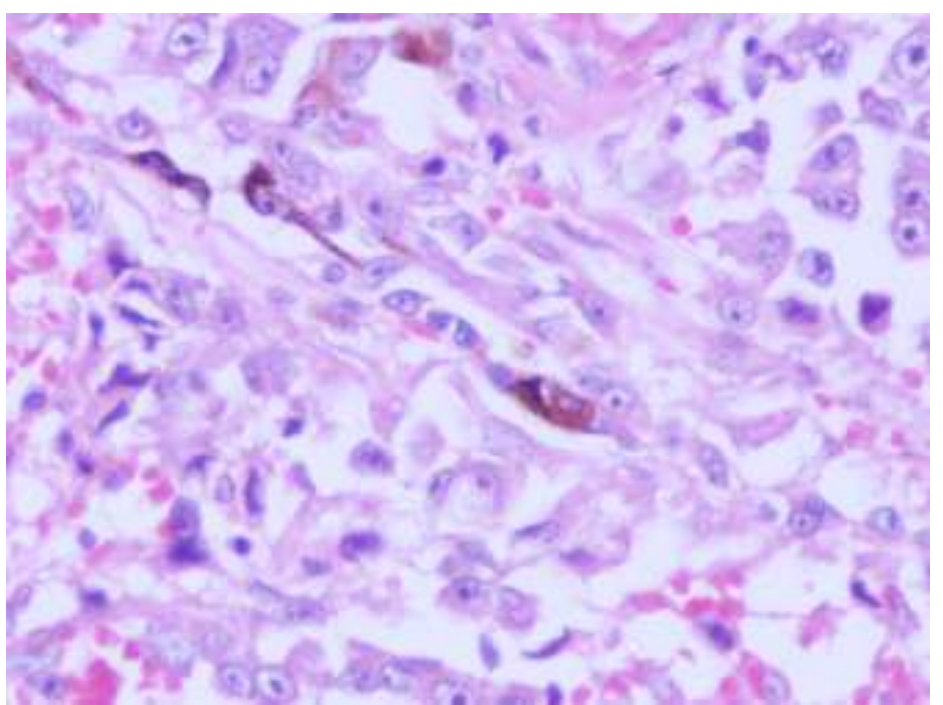

Fig-7: Epithelioid to spindled tumor cells with clear to pale cytoplasm, prominent nucleoli, and cytoplasmic melanin pigment (hematoxylin-eosin, original magnification X400). 


\section{Immunohistochemistry}

A panel of immunohistochemical stains for diagnostic workup were performed include S100 protein, HMB45, Melan-A, EMA, GFAP, CKPAN, Ki67. The neoplastic cells were positive for S100 protein with focal weak nuclear and cytoplasmic staining pattern. HMB45 showed focal strong cytoplasmic immunoreactivity (Fig.8). Melan-A stain is strongly expressed in the majority of neoplastic cells (Fig.9). Stains for CKPAN and EMA were completely negative. Ki-67 labelling index shows positive staining in 8 to 12 $\%$ of nuclei. Glial fibrillary acidic protein stain (GFAP) is negative in the melanoma cells, while it is strongly expressed in the adjacent non-neoplastic cortical brain tissue.

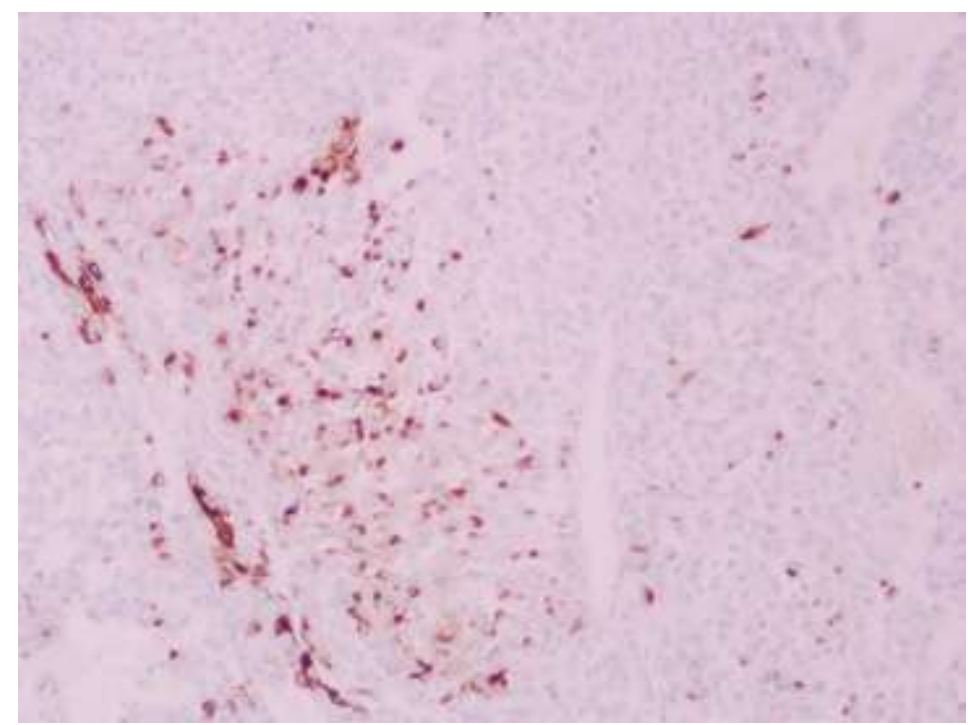

Figu-8: $H M B-45$ immunohistochemical stain shows focal strong positivity in the neoplastic melanoma cells (original magnification X200)

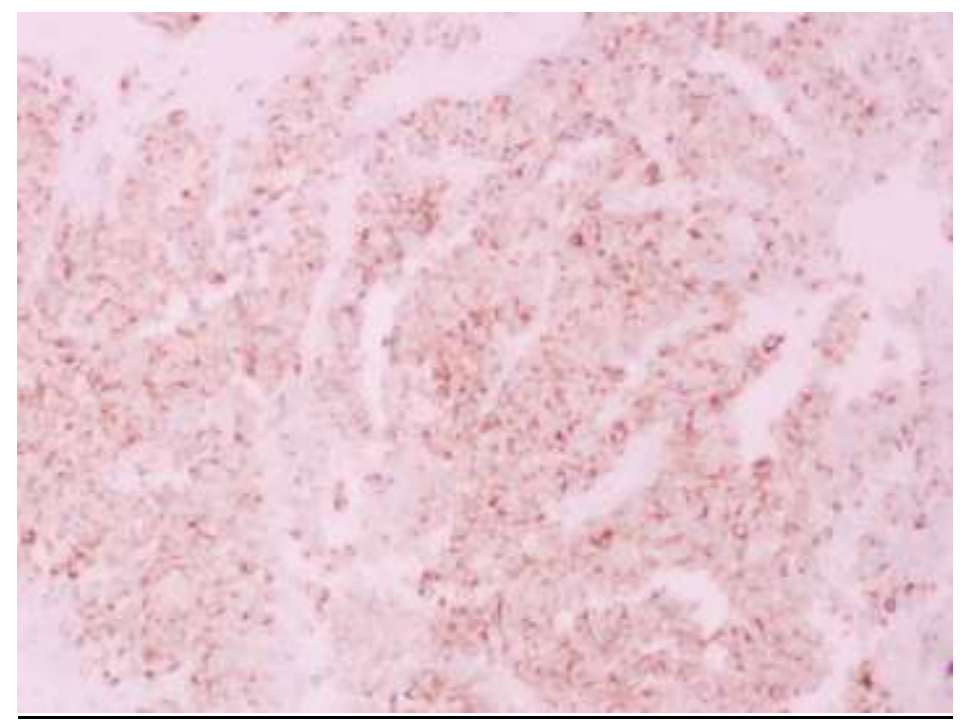

Fig-9: Melan-A immunohistochemical stain is diffusely expressed in the neoplastic melanoma cells (original magnification X200)

\section{Clinical management}

The patient admitted to the intensive care unit. High ICP management and mannitol therapy were initiated. After failure of the therapy, his pupil in the right side started to dilate. With this rapid deterioration in his condition, emergency surgery was performed. Left pterional craniotomy and complete resection of the lesion was done. Physical character of lesion was dark brown in color. The challenge of resection was to preserve the speech area, which is located in distance of
4-5 cm from left temporal lobe tip. Part of the lesion was reaching the base of the frontal lobe which was approached by pterional craniotomy after splitting the Sylvian fissure. Dural attachment of lesion was completely excised and repaired with dura graft upon closure. Patient discharged to home after 4 days staying in hospital with follow up CT brain. The patient came for follow-up in good condition with remarkable improving of dysarthria. Further work up was performed such as referral to radiation oncology 
department and dermatology consultation to rule out distant metastasis and to exclude the possibility of metastatic melanoma to the CNS. The workup was negative for any skin lesions, mucosal lesions, and for distant metastasis, too.

\section{DISCUSSION}

The WHO classified primary melanocytic neoplasms of the CNS into four categories based on the growth pattern. The diffuse form, which can be either benign (meningeal melanocytosis) or malignant (meningeal melanomatosis). The localized massforming tumors with benign features are termed (meningeal melanocytoma) whereas those with malignant features are called (meningeal melanoma) [5]. Metastatic melanomas outnumber primary CNS melanomas, which account only for $1 \%$ of intracranial melanomas \& $0.1 \%$ of all intracranial tumors [6]. Thus, the diagnosis of primary CNS melanoma should be done after a complete and extensive workup to exclude metastasis from primary extra-cranial melanoma.

\section{Clinical features}

Primary CNS (meningeal) melanoma is a rare tumor with an incidence of 0.005 cases per 100000 populations per year [7]. Based on the review of previously reported thirty-four cases in the literature, it appears that the tumor affects a wide age range, 5 to 76 years (mean age: 38 years) $[8,9]$.

There is a slight male predominance $(\mathrm{M}: \mathrm{F}=$ $2.2: 1.2)$. The patients usually present with rapidly evolving focal neurological symptoms related to tumor location. Most of the patients presented with headache, nausea, and vomiting. Other symptoms include blurred vision, dizziness, convulsion, mentality change, and ataxia. In all the reported cases, there was no evidence of extra neural cutaneous or mucosal melanomas. One of the cases was associated a contralateral nevus of Ota [10].

\section{Radiologic features \& gross pathology}

Meningeal melanomas are rapidly growing, usually dural-based solid tumors, which can occur anywhere in the neuraxis. They show a predilection for the ventral aspect of the spinal cord and posterior fossa [5]. About 70 to $80 \%$ of tumors reported in the literature were located in the left hemisphere. The left temporal lobe and left frontal lobe are most frequently affected. About $15 \%$ of tumors were located in the right hemisphere and the remaining was present in the midline (three cases in the pineal region \& one case in the medulla oblongata). Radiologically, four different MRI presentations have been proposed for meningeal melanoma based on tumor melanin content. These include: (1) melanoma, characterized by hyper-intense T1 image and hypo- or iso-intense on T2 image; (2) non-melanoma, characterized by hypo- or iso-intense on T1 image and hyper- or iso-intense on T2 image; (3) uncertain or mixed, characterized by mixed MRI signal; and (4) hemorrhagic lesions, characterized by different stage of hemorrhage on MRI. The melanoma and hemorrhagic-type lesions account for approximately $70 \%$ of malignant intracranial melanomas [6]. Due to the rapid growth rate of these tumors, peri-tumoral vasogenic edema is usually noted.

Grossly, meningeal melanomas are solitary, solid, variably pigmented tumors, which can be gray, brown, or black. Gross invasion of the adjacent brain parenchyma is usually evident [5].

\section{Histological \& immunohistochemical features}

Microscopic features of primary CNS melanoma parallel those of its systemic counterpart. Tumors are usually cellular and comprise of solid sheets, loose nests, or fascicles of atypical cells with focal papillary growth pattern. Individual tumor cells are spindle or polygonal with enlarged round to oval nuclei, high nuclear to cytoplasmic ratio, eosinophilic macronucleoli, and significant pleomorphism. The cytoplasm is pale to eosinophilic with variable melanin pigment. Tumors are mitotically active (mean, 5.7/10HPFs) [11]. Coagulative tumor necrosis and unequivocal brain invasion are usually seen. Some tumors exhibit large bizarre highly pleomorphic nuclei.

Immunohistochemistry, primary CNS melanomas are usually diffusely positive for markers of melanocytic differentiation, including S100 protein, Melan-A (MART-1), HMB-45, Micro-ophthalmia transcription factor, and tyrosinase [12]. Tumors are usually negative for Cytokeratins, EMA, and GFAP, which is of diagnostic importance to exclude tumors in the differential diagnosis. The Ki-67/MIB-1 labeling index is always high (mean, 8.1\%) [13].

\section{Genetic profile}

Primary CNS melanomas are genetically distinct from cutaneous melanomas. They show high frequency of GNAQ (71\%) and GNA11 (12\%) gene mutations. Uncommonly, primary CNS melanomas may show inactivating mutation of $B A P 1$ gene and loss of chromosome 3 . These two genetic alterations could represent potential prognostic markers in primary CNS melanomas. Loss of BAP1 nuclear staining, which can be detected by immunohistochemistry serves as an adverse prognostic marker. In rare, mainly pediatric cases of primary CNS melanomas, NRAS mutation have been reported. It is found that primary CNS melanomas share several genetic alterations with primary uveal melanoma; particularly GNAQ (45-47\%) and GNA11 (32-44\%) gene mutations. Mutations in genes typically seen in cutaneous melanomas such as $B R A F, K I T$, and $T E R T$ promoter are extremely rare and if they are found should raise the possibility of metastatic melanoma from cutaneous origin [13]. Detection of genetic alterations particularly DNA-methylation profiles, copy-number variants, and gene mutations are helpful 
in distinguishing between different types of melanotic nervous system tumors [14].

\section{Differential Diagnosis}

The differential diagnosis for a pigmented lesion of the CNS includes three categories: (1) primary melanocytic neoplasms of the CNS, (2) metastatic melanoma to the CNS, and (3) melanin-containing variants of other intracranial tumors. The later are rare and include meningiomas, melanotic schwannomas, medulloblastomas, some gliomas, cranyopharyngioma, melanotic neuroectodermal tumor of infancy, and melanocytes in teratoma [15]. It is important to differentiate primary melanin containing lesions of the CNS from metastatic melanoma, because these lesions require a different patient workup and management. The absence of a known primary extracranial melanoma helps in the differential diagnosis; however, an occult primary lesion outside the CNS must be excluded.

Comprehensive patient workup including proper clinical evaluation, radiological findings, coupled with pathological assessment with or without testing for genetic alterations lead to appropriate patient diagnosis and management.

\section{REFRENCES}

1. Crasto, S. G., Soffietti, R., Bradac, G. B., \& Boldorini, R. (2001). Primitive cerebral melanoma: case report and review of the literature. Surgical neurology, 55(3), 163-168.

2. Suzuki, T., Yasumoto, Y., Kumami, K., Matsumura, K., Kumami, M., Mochizuki, M., \& Kojima, H. (2001). Primary pineal melanocytic tumor: Case report. Journal of neurosurgery, 94(3), 523-527.

3. Virchow, R. (1859). Pigment und diffuse Melanose der Arachnoides. Archiv für pathologische Anatomie und Physiologie und für klinische Medicin, 16(1-2), 180-182.

4. Pappenheim, E., \& Bhattacharji, S. K. (1962). Primary melanoma of the central nervous system: Clinical-pathological report of a case, with survey and discussion of the literature. Archives of neurology, 7(2), 101-113.

5. Cavenee, W. K., Louis, D. N., Ohgaki, H., \& Wiestler, O. D. (Eds.). (2007). WHO classification of tumours of the central nervous system (Vol. 1). WHO Regional Office Europe.
6. Lee, P. H., Wang, L. C., \& Lee, E. J. (2017). Primary intracranial melanoma. Journal of Cancer Research and Practice, 4(1), 23-26.

7. Helseth, A., Helseth, E., \& Unsgaard, G. (1989). Primary meningeal melanoma. Acta Oncologica, 28(1), 103-104.

8. Nicolaides, P., Newton, R. W., \& Kelsey, A. (1995). Primary malignant melanoma of meninges: atypical presentation of subacute meningitis. Pediatric neurology, 12(2), 172-174.

9. Rovlias, A., Siakavella, M., \& Fragakis, G. (2015). Primary supratentorial malignant melanoma: a case report with long survival. Journal of the Neurological Sciences, 357, e433.

10. Guthikonda, B., Ahmed, O., Buckleair, L. J., Goodman, J. C., Powell, S. Z., \& Yoshor, D. (2015). Primary CNS malignant melanoma associated with a contralateral nevus of Ota in an African-American female: Unique Case Report. Cancer and Clinical Oncology, 4.

11. Brat, D. J., Giannini, C., Scheithauer, B. W., \& Burger, P. C. (1999). Primary melanocytic neoplasms of the central nervous system. The American journal of surgical pathology,23(7), 745.

12. Jaiswal, S. (2016). Role of immunohistochemistry in the diagnosis of central nervous system tumors.

13. van de Nes, J., Gessi, M., Sucker, A., Möller, I., Stiller, M., Horn, S., ... \& Zimmer, L. (2016). Targeted next generation sequencing reveals unique mutation profile of primary melanocytic tumors of the central nervous system. Journal of neuro-oncology, 127(3), 435-444.

14. Koelsche, C., Hovestadt, V., Jones, D. T., Capper, D., Sturm, D., Sahm, F., Schrimpf, D., Adeberg, S., Böhmer, K., Hagenlocher, C., Mechtersheimer, G., Kohlhof, P., Mühleisen, H., Beschorner, R., Hartmann, C., Braczynski, A. K., Mittelbronn, M., Buslei, R., Becker, A., Grote, A., Reuss, D. E. (2015). Melanotic tumors of the nervous system are characterized by distinct mutational, chromosomal and epigenomic profiles. Brain pathology (Zurich, Switzerland), 25(2), 202-208.

15. Smith, A. B., Rushing, E. J., \& Smirniotopoulos, J. G. (2009). Pigmented lesions of the central nervous system: radiologic-pathologic correlation. Radiographics: a review publication of the Radiological Society of North America, Inc, 29(5), 1503-1524. 